\title{
The research of intersection traffic violation system
}

\author{
NanYan Liư ${ }^{1, a}$, Peng $\mathrm{Hu}^{2, \mathrm{~b}}$ \\ ${ }^{1}$ School of Xi'an University of Science and Technology, Xi'an 710054, China; \\ ${ }^{2}$ School of Xi'an University of Science and Technology, Xi'an 710054, China. \\ a513318452@qq.com, b337512784@qq.com
}

Keywords: Video foreground separation, Lane detection, Hough transform, License plate location.

\begin{abstract}
Nowadays most of the traffic system of the General research focuses on key points, such as vehicle contour segmentation precision, the effectiveness of vehicle information, research on intersection traffic violation system there are still big problems. By using CodeBook[2] based on time series algorithm to real-time reconstruction of the video in the background, also add texture feature for foreground options, the better off the vehicle with the outline. Lane detection based on the traditional Hough transform, fine filters combined with the outline of the image itself, more precisely isolate a lane. Access to vehicle information, using the SVM classifier in machine learning methods $[3,4]$ to get the multi-lane vehicle information, reduces the false detection of license plate location in complicated environment, achieving a key part.
\end{abstract}

\section{Introduction}

Intersection traffic violation system is an important part of intelligent transportation system, this system based on image processing, in view of the intersection signal control, to test the motor vehicles cross the line. Uses the camera to cover multiple lanes, the yellow line of motor vehicles, parking gets crossed the line in violation of information. Statistics in the context of big data analysis and forecasting needs and study of parallel computing, image processing of the acquired data and visualization is an important part of intelligent control. Intersection traffic violation system for realtime video processing, operation, extraction of each frame image of vehicle information, in order to obtain the number of cars, vehicles' whereabouts, and finally achieve the goal of intelligent transportation. Intersection traffic violation system research part is to real-time video streaming background reconstruction, fine separated out of the vehicles contours, get vehicle information, to judge the vehicles cross the line. Through much of the existing research, improve its methods of detection, base preparation for realization of intelligent transportation systems.

\section{Video foreground separation}

For a fixed camera captured video of road cars, video stream background separation is commonly used by video frame data for average or background of Gaussian probability to form a close, such as Gaussian model[1] method. Find a good of algorithm can most close current background data established background model is research of key, most background built die research are exists is big pixel value errors, led to separation of effect does not good. Feng Pu[5], people using vehicles of texture features established mixed Gaussian background model algorithm, the method to establish the background absorption of the gaussian background modeling advantage, while on vehicles pixel has better of judge, better of separation out has vehicles foreground. CodeBook algorithm is to obtain a time series data for each pixel, through these data for training to establish a code of this model, for detection of video frames, use the established model for judging, data within a certain threshold as a background, or as a foreground data. At the same time to certain dynamic updating of the model is set up before, thus image background in one day to have a real-time modeling of separating out the clear image of vehicles.

Due to intersection traffic violation system all-weather work, one days in the of light changes differences is big. Gaussian mixture model in real time on the background pixel value to be inferior 
to the CodeBook modeling, but CodeBook model of vehicle pixels with similar pavement of pixels, there will be a miscalculation. So intersection traffic violation system integration of both, first get CodeBook algorithm results, then the pixel to determine the result, if a pixel belongs to the texture of the body area, this area is selected to obtain the best results, results such as "Fig.1 (2)". Intersection traffic violation system will ultimately choose vehicle contour range to determine if it is covered with the lane line. And this system adopt the method of rotating quadrilateral approximation to get the vehicle outline border, to determine whether there are illegal, contour boundary approximation results as shown in Fig.1(3).

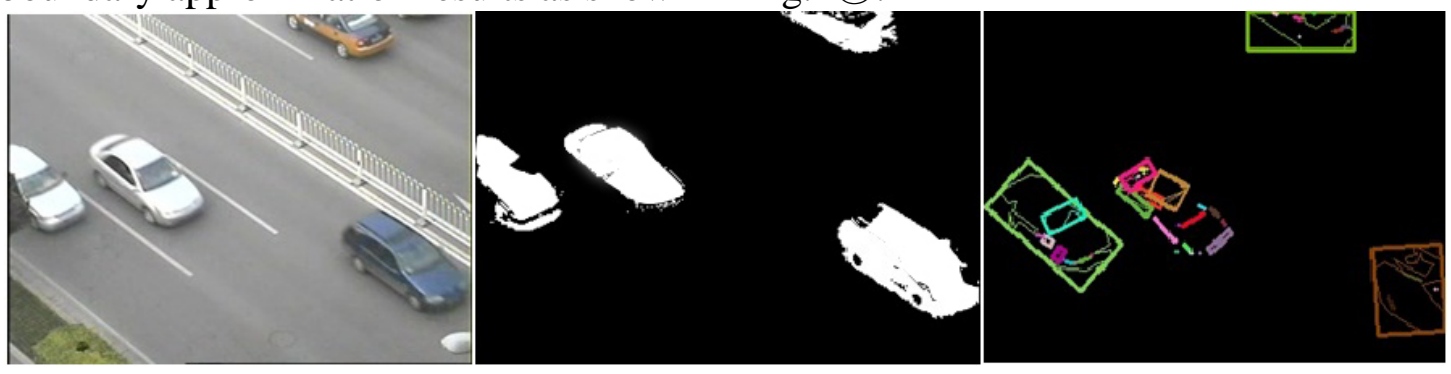

Fig. 1 (1)Single frame image(2)foreground Image(3)Contour approximation image

CodeBook algorithm for each pixel of the current image to build a code book (CB) structure, each CodeBook structure consists of several code symbol $(\mathrm{CW}) . \mathrm{CW}=\{$ High, Low, max, min, t_last, stale $\}$, Algorithms in use process, element structure to the $\mathrm{CW}=$ \{High, Low, Max, min, $\mathrm{t} \_$last, stale, modelLike , by adding an element: texture similarity. Texture similarity around $5 * 5$ block image in pixels of the mean, standard deviation, maximum, minimum, the current pixel values of five feature weighting computation. $C B=\{C W 1, C W 2, \ldots, C W n, t\}$. In the experiment, the video stream is greater than two minutes code learning, set up the Basic code of the model and texture statistics of the vehicle, and must be quantified. Again using established good of CB for movement target detection, background of range upper and lower territories for minMod and maxMod, for current stay detection image of a pixel I(x,y), traverse it corresponds to pixel background model CB in the of each a CW, if exists a CW, makes $\mathrm{I}(\mathrm{x}, \mathrm{y})<\max +\operatorname{maxMod}$ and $\mathrm{I}(\mathrm{x}, \mathrm{y})>\min -\min M o d$, and texture similar degrees is less than a value, $\mathrm{I}(\mathrm{x}, \mathrm{y})$ was judge for background, or was judge for foreground.

\section{Traffic lane detection}

For the traffic lane detection, many intelligent driving research is the real-time detection of lane line [6], the case of the route is not clear or the line marked messy, resulting in large disturbance and inaccurate. We do not need to obtain the route information in real time for the intersection violation system, the traditional method of route detection to get the information of the line with Hough transform, such as Yang Xining et al by Hough transform to establish angle constraints for positioning the lane [7]. Based on the improvement of Hough transform, this system will "AND" the line detection image with the contour image detected by the original image Canny operator, which can improve the accuracy to a certain extent, as shown in [Fig.2], [Fig. 3] and [Fig. 4]. In the experiment ,before using the Hough transform, the pixel value of the whole image is smaller than 210, which will widen the contrast between the white line and the surrounding interfering object, and then use the line detection and edge detection. This experiment on the route across the upper boundary and the lower boundary of image in the straight line threshold selection, the image size is $640 * 360$, and the number of points on the same straight line need to reach 150, which can effectively filter the Interference. 


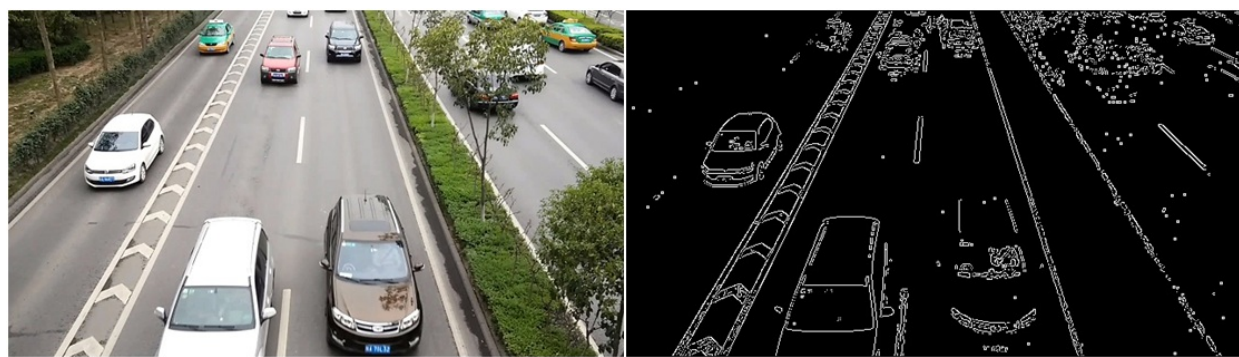

Fig. 2 The original image and the contour image

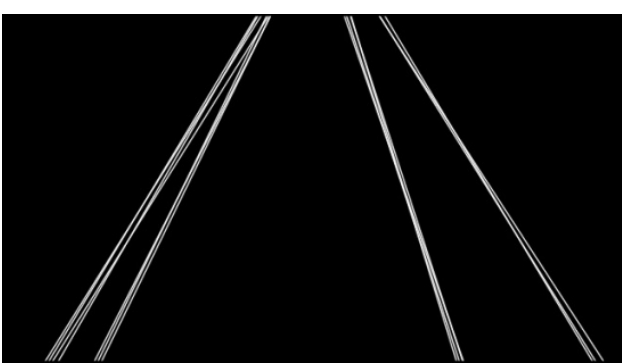

Fig. 3 Hough transform for line detection images

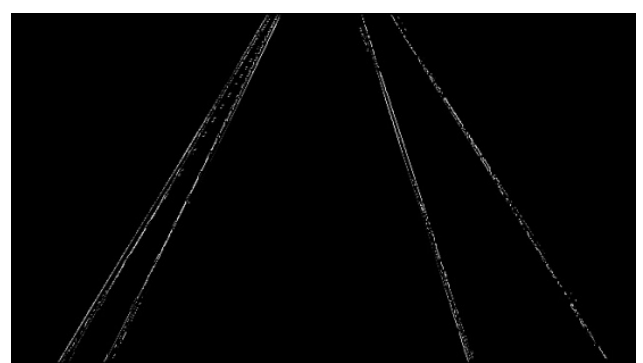

Fig. 4 "And" transform the image

\section{License plate location research}

At present, the method of license plate location include the method based on edge detection method ,based on gray level variation characteristics, method of license plate based on color features and neural network learning machines and other methods to obtain the license plate,the license plate recognition based on image processing has been widely applied to the license plate information acquisition system, the express highway toll collection, these scenes have their common characteristic is the vehicle location fixed, camera and vehicle distance fixed, singlechannel vehicles through the detection, the effect is relatively high. However, if the multi -Lane vehicle, the camera to capture the picture is more extensive, the above mentioned method is not applicable.

Therefore, in order to realize multi-lane vehicles and capture a wide range of images, this system uses the HOG method[8] with the SVM classifier to locate the license plate, obtained the positive samples by fine-cutting the images, non plate region as negative samples to improve the classification accuracy, and then treat the detected image multi scale detection, cropping of the positive sample data image shown in Fig.5, this method in the study of pedestrian detection also has a high success rate. This system use this method also can get a higher detection rate, but also to track the role of the detection results shown in Fig.6, From Fig.6 can clearly see the license plate location of multiple vehicles. At the same time, the system can obtain the complete contour of the license plate by using the edge detection method with higher accuracy rate for the illegal vehicle information detection.

Classifier is defined as the $\arg \max _{\mathrm{w}, \mathrm{b}}\left\{\min _{\mathrm{n}}\left(\right.\right.$ lable $\left.\left.\cdot\left(\mathrm{w}^{\mathrm{T}} \mathrm{x}+\mathrm{b}\right)\right) \cdot \frac{1}{\|\mathrm{w}\|}\right\}$.

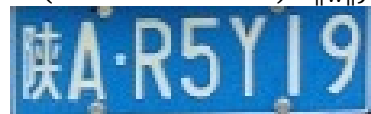

Fig. 5 The positive sample Image 


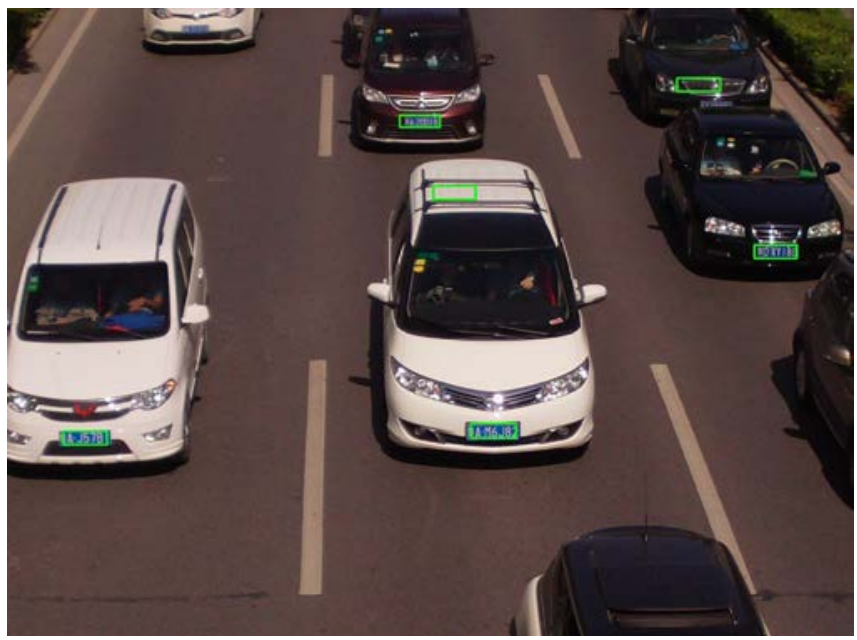

Fig. 6 Multi-lane vehicle detection results

\section{Summary}

Intersection traffic violation system's main problem is that the video streaming separation of high integrity, the accuracy of the information above the line detection and vehicle. Through the integrated use of existing technologies, to get the best results. But its feasibility is still a lot of room for improvement, such as vehicle shadow removal, video night lights and other factors.

\section{References}

[1] Stauffer C, Grimson W E L. Adaptive Background Mixture Models for Real-Time Tracking[J]. Proc Cvpr, 1999, 2:2246.

[2] [US] Bradski G. Learning OpenCV [J]. 2009.

[3] Bartlett M S, Movellan J R, Sejnowski T J. Face recognition by independent component analysis[J]. IEEE Transactions on Neural Networks, 2002, 13(6):1450-64. [4] http://blog.csdn.net/liulina603/article/details/8291093.

[5] FENG Pu, LI Yuhui, LI Bo, YOU Xuchen. Research on Gaussian Mixture Background Modeling Algorithm Based on Texture Feature[J]. COMPUTER TECHNOLOGY AND DEVELOPMENT, 2016, 26(5).

[6] Michalopoulos P G. Vehicle detection video through image processing: the Autoscope system[J]. IEEE Transactions on Vehicular Technology, 1991, 40(1):21-29.

[7] Yang Xining, Duan Jianmin, Gao Dezhi, Zheng Banggui. Research on Lane Detection Based on Improved Hough Transform[J]. Computer Measurement \& Control, 2010, 18(2):292-294.

[8] Viola P, Jones M. Rapid object detection using a boosted cascade of simple features[J]. Proc Cvpr, 2001, 1:511. 